Rev. Élev. Méd. vét. Pays trop., 1977, 30 (4) : 383-386.

\title{
Note sur quelques complications tardives observées à la suite de l'anesthésie du zébu (bos indicus) par le couple « Immobilon - Revivon » ND
}

\author{
par M. GRANGE et J. P. DENIS (**)
}

\begin{abstract}
RESSUMÉ
Les auteurs décrivent des accidents tardifs observés chez des zébus Gobra à robe claire expérimentalement traités avec un neuroleptanalgésique (Immobilon) et son antagoniste (Revivon). lis attribuent ces phénomènes à un processus de photosensibilisation induit par le dérivé de la phénothiazine contenu dans l'immobilon. Ils en discutent les raisons, proposent un traitement efficace en insistant sur la prudence dont il convient de faire preuve dans leur utilisation chez des bovins à robe claire, par grand soleil et forte chaleur.
\end{abstract}

\section{INTRODUCTION}

Le Centre de Recherches Zootechniques de Dahra, situé en plein cœur du Sénégal en zone sahélienne, a pour vocation principale l'étude de la race zébu sénégalaise Gobra. Ces animaux, caractérisés par un pelage très clair, ont parfois un caractère farouche, voire agressif qui rend difficiles sinon dangereuses leur approche et leur contention.

Les résultats obtenus dans diverses régions pour tranquilliser ou très provisoirement anesthésier de grands animaux domestiques à l'aide de neuroleptanalgésiques et surtout de leurs antagonistes pour hâter leur réveil, nous ont conduit à essayer le couple Immobilon-Revivon sur des sujets du Centre.

Les résultats, favorables, obtenus auraient pu être passés sous silence si l'utilisation de ces produits dans les conditions ambiantes précitées

(*) «Immobilon-Revivon » large animal, laboratoires Reckitt et Colman (Angleterre).

(**) Laboratoire national de l'Elevage, B. P. 2057 , Dakar-Hann (Sénégal). n'avaient conduit à des accidents, que nous pensons pouvoir être rattachés à des réactions de sensibilisation, si graves qu'il nous a paru opportun d'en faire état dans cette note au profit de ceux qui pourraient être appelés à les utiliser.

\section{MATERIEL ET METHODES}

\section{A. Matériel}

a) Neuroleptanalgésique et son antagoniste

L'association Neuroleptanalgésique «Immobilon» et son antagoniste le «Revivon» est utilisée depuis quelques années chez les animaux domestiques. Pour les espèces de grand format (bovins et cheval en particulier), ces produits sont commercialisés sous la forme d'un coffret contenant 2 flacons de $10,5 \mathrm{ml}$, chaque millilitre renfermant :

- pour «l'Immobilon » large animal 2,45 mg d'Etorphine (ou M-99) - Analgésique "Morphinique » de synthèse doué d'une activité analgésique environ 10000 fois supérieure à celle de la Morphine lorsqu'on la teste chez 
la souris $-10 \mathrm{mg}$ de maléate d'acépromazine en solution salée de chlorocrésol ;

- la solution antagoniste ou "Revivon» large animal renferme $3 \mathrm{mg}$ de chlorhydrate de Diprenorphine (Antagoniste «spécifique » de l'Etorphine), en solution dans le bleu de Méthylène et le chlorocrésol (solution bleutée permettant de la distinguer aisément de la précédente). L'effet antagoniste assurant un " réveil » rapide de l'animal est obtenu par injection d'un volume de «Revivon» égal à celui d'"Immobilon » préalablement injecté.

\section{b) Animal}

Les animaux sont des zébus de race Gobra (zébu Peulh sénégalais). Il s'agit de 4 mâles de réforme du C.R.Z. de Dahra âgés de 3 ans environ dont les poids étaient les suivants :

$$
\begin{array}{llll}
\mathrm{n}^{\circ} & 2916 \ldots \ldots \ldots & 325 \mathrm{~kg} \\
\mathrm{n}^{\circ} & 2964 \ldots \ldots \ldots & 333 \mathrm{~kg} \\
\mathrm{n}^{\circ} & 2965 \ldots \ldots \ldots & 303 \mathrm{~kg} \\
\mathrm{n}^{\circ} & 2968 \ldots \ldots \ldots & 295 \mathrm{~kg}
\end{array}
$$

au moment du $1^{\text {er }}$ essai et $303 \mathrm{~kg}$ pour le $\mathrm{n}^{0} 2964$ lors du second essai effectué un mois plus tard.

\section{B. Méthode}

Les injections ont été faites par la voie intraveineuse aux doses de $1 \mathrm{ml} / 100 \mathrm{~kg}$ ( ${ }^{\circ / 2} 2964$ et 2968$), 0,75 \mathrm{ml} / 100 \mathrm{~kg}\left(\mathrm{n}^{\circ} 2965\right)$ et $0,50 \mathrm{ml} /$ $100 \mathrm{~kg}$ (2916).

\section{RESULTATS}

\section{A. Déroulement des essais}

Le premier essai a été effectué au mois de juin (température extérieure supérieure à $40{ }^{\circ} \mathrm{C}$ sous abri) en 2 temps : tout d'abord dans l'après-midi pour le $\mathrm{n}^{\circ} 2916$ puis le lendemain matin pour les 3 autres taurillons. Enfin le $n^{\circ} 2964$ a été retraité un mois plus tard et vasectomisé à cette occasion.

Aux diverses doses employées, le décubitus latéral a été obtenu en moyenne $10 \mathrm{mn}$ après l'injection d'《Immobilon »; la sédation de l'animal est correcte bien que persistent cependant des mouvements de défense. Chez l'animal vasectomisé, ces mouvements brusques ont été gênants pour le déroulement de l'intervention et une anesthésie locale a été nécessaire pour le deuxième testicule.

Pour les n² 2916, 2964 et 2968 , le « réveil 》 induit par injection I. V. de "Revivon» a été très rapide ( $2 \mathrm{mn}$ environ), suivi immédiatement du relever de l'animal qui retrouve toute son agressivité et cherche à s'alimenter sur le champ. Le $\mathrm{n}^{\circ} 2965$ a présenté un réveil plus tardif ( $8 \mathrm{mn}$ environ).

Après le réveil, les animaux présentent une légère ataxie locomotrice pendant une $1 / 2 \mathrm{~h}$ à $1 \mathrm{~h}$.

Le lendemain matin, l'animal 2916 anesthésié l'après-midi précédente n'a pas d'appétit et sa démarche est un peu raide; il présente des difficultés respiratoires accompagnées d'hyperthermie $\left(40^{\circ} 2\right)$. Vers $16 \mathrm{~h}$, on observe de la météorisation, une respiration franchement dyspnéique, une salivation abondante ainsi que de l'cedème crépitant au niveau de la ganache et du fourreau. L'odème gagne le chanfrein, le tissu sus-orbitaire et devient général au niveau de la tête; on note des trémulations violentes du tenseur du Fascia lata et des muscles de la cuisse.

Vers $19 \mathrm{~h}$, l'animal chute brusquement, mort. A l'autopsie, l'ensemble du tissu conjonctif sous-cutané est odématié ; les poumons sont très odémateux, le cœur est vide de sang.

Une douzaine d'heures après l'injection d' « Immobilon » suivie du réveil par son antagoniste, les trois autres animaux, traités le matin même, présentent des signes prémonitoires analogues.

Un traitement à l'aide de Prométhazine (*) a été tenté sans résultats sur l'animal $n^{\circ} 2916$ (250 $\mathrm{mg}$ en I. V.) et avec succès chez les trois autres ( $250 \mathrm{mg}$ en I. M.) dont les troubles ont rapidement régressé.

\section{DISCUSSION}

Les injections ont été effectuées en plein soleil au mois de juin où la température sous abri dépasse $40^{\circ} \mathrm{C}$. Les animaux utilisés ont une robe blanche, froment très clair pour l'un d'entre eux ( $\left.\mathrm{n}^{\circ} 2964\right)$.

(*) Phénergan ND. 
Or, l'association Neuroleptanalgésique « Immobilon * «large animal » est un mélange d'Etorphine et d'Acépromazine qui est un dérivé de la série des Phénothiazines. Pour expliquer les accidents observés, il semble bien que l'on doive se tourner vers un phénomène de violente photosensibilisation provoquée par la présence de ce dérivé phénothiazique dont l'injection a déclenché chez les animaux ainsi traités une réaction "allergique 》 grave de type urticarien, sans doute associée à des déséquilibres thermorégulateurs centraux.

Rappelons que l'utilisation de l'association analgésique majeur (Etorphine ou Fentanyl) + Acétylpromazine pour la capture des grands mammifères sauvages (Antilopes et buffles notamment) a permis d'observer des accidents voisins (photosensibilisation et déséquilibres thermorégulateurs) chez les animaux à robe sombre immobilisés en plein soleil $(4,3,5)$, dont la pathogénie peut être partiellement rattachée à l'action de ce neuroleptique sur les centres thermorégulateurs de l'hypothalamus à laquelle s'ajoute celle du soleil et d'une température extérieure très élevée.

L'animal $\pi^{\circ} 2916$, bien qu'ayant reçu la dose la plus faible, a succombé probablement parce qu'anesthésié aux heures les plus chaudes de la journée mais surtout parce que traité trop tardivement.

Le $n^{\circ} 2964$ lors du second essai a reçu une injection de phénergan préventive et n'a montré aucun trouble ultérieur.

\section{CONCLUSION}

L'utilisation du couple «Immobilon-Revivon large animal » peut s'adresser à des animaux particulièrement farouches dont l'approche et la contention sont difficiles. Les résultats semblent valables et constants tant pour la sédation et l'analgésie obtenues que pour la rapidité du « réveil » induit par l'antagoniste. On doit cependant dans la mesure du possible éviter l'emploi de ces produits chez des animaux zébus à robe très claire exposés en plein soleil et lorsque la température extérieure est élevée; on doit de même prévoir une intervention éventuelle à l'aide de phénergan.

Il est évident que ces données sont fragmentaires et qu'une expérimentation plus complète serait nécessaire pour préciser la pathogénie des accidents observés. Nous avons cependant estimé utile d'attirer l'attention des utilisateurs éventuels de ces produits sur la possibilité de ce type d'accident.

\section{SUMMARY}

Note on some low accidents observated after zebu cattle anaesthesia by Immobilon-Revivon ND

«Immobilon " large animal can be used successfully to approach and immobilize shy and agressive animal. Through the results are quite valid and constant one has to take care about photosensitization accidents when utilizing the mixture on zebu (Bos indicus) animal, particularly white-skinned an1mals immobilized in the sun when the outside temperature is elevated.

\section{RESUMEN}

Nota sobre algunas complicaciones tardias observadas después de la anestesia del cebú (Bos indicus) con el conjunto « Immobilon-Revivon »

Los autores describen accidentes tardios observados en cebues Gobra de pelo claro experimentalmente tratados con un neuroleptanalgésico (Immobilon) y su antagonista (Reviyon). Atribuyen dichos fenómenos a un proceso de fotosensibilización inducido por el derivado de la fenotiazina contenido en el Immobilon. Discuten las razones de eso, proponen un tratamiento eficaz al insistir sobre la prudencia que se necesita observar en cuanto a su utilización en los bovinos de pelo claro, cuando hay un sol y un calor importantes. 


\section{BIBLIOGRAPHIE}

1. GRANGE (M.). Immobilisation des mammifères sauvages et apports de l'Etorphine en neuroleptanalgésie vétérinaire. Thèse Doct. vét. Lyon, $1972, \mathrm{n}^{\circ} 80$, 181 p., 254 réf.

2. GRANGE (M.), BOIVIN (R.), FARGES (J. P.) Nouvelle technique de neuroleptanalgésie réversible chez le chien. Utilisation de l'étorphine et de son antagoniste la diprénorphine. Rev. Méd. vét., 1973, 124 (7) : 899-908.

3. HARTHOORN (A. M.). The use of a new Oripa- vine derivative for restraint of domestic hoofed animals. J. S. afr. vet. med. Ass., 1965, 36 (1) : 45-50.

4. HARTHOORN (A. M.). Restraint of undomesticated animals. J. Am. vet. Assoc., 1966, 149 (7): 875-880.

5. PIENAAR (U. de V.). Recent advances in the field immobilization and restraint of wild Ungulates in South African national Parks. Antwerp. Zoo. J., $1968,48: 17-38$. 